\title{
Recursos fisioterapêuticos aplicados no trabalho de parto natural humanizado: uma revisão bibliográfica
}

\author{
Physical therapy resources applied in humanized natural labor: a bibliographic review \\ Recursos fisioterapéuticos aplicados en el trabajo natural humanizado: una revisión \\ bibliográfica
}

Carolline Bittencourt da Cruz ${ }^{1}$, Carla Lorena de Araujo Costa ${ }^{1}$, Raiane Santos de Brito ${ }^{1}$, Luana de Jesus Reis ${ }^{1}$, Lília Tatiane dos Santos Sá1, Izadora Santos Paciência ${ }^{1 *}$.

\section{RESUMO}

Objetivo: Discutir as contribuições dos recursos fisioterapêuticos aplicado no trabalho de parto natural humanizado. Métodos: Revisão de literatura pesquisada no horizonte temporal de 2009 a 2019 nas bases eletrônicas de dados BVS, PubMed, SciELO, PEDro, e Google Acadêmico. O processo de análise dos artigos selecionados deu-se por meio da leitura exploratória e detalhada de títulos, resumos e dos resultados das pesquisas, onde se buscaram as contribuições dos recursos fisioterapêuticos aplicados no trabalho de parto natural humanizado. Resultados: Dentre as opções dos recursos fisioterapêuticos os mais comumente usados para a redução do quadro álgico no processo de trabalho de parto natural humanizado são: exercícios respiratórios, massoterapia, termoterapia, alternância de posições, exercícios com o auxílio da bola suíça e eletroterapia. Considerações Finais: Os recursos fisioterapêuticos utilizados oferecem resultados positivos na redução do quadro álgico assim como na duração do tempo do trabalho de parto, tornando-o mais ativo, humanizado e satisfatório para a parturiente.

Palavras-chave: Fisioterapia, Parto humanizado, Saúde da mulher.

\begin{abstract}
Objective: Discuss the contributions of physical therapy resources applied in humanized natural labor. Methods: Literature review researched on the time horizon from 2009 to 2019 in the electronic databases BVS, PubMed, SciELO, PEDro, and Google Scholar. The analysis process of the selected articles took place through the exploratory and detailed reading of titles, abstracts and research results, where the physiotherapeutic resources applied in humanized natural labor were sought. Results: Among the options of physiotherapeutic resources the most commonly used to reduce pain in the humanized natural labor process are breathing exercises, massage therapy, thermotherapy, position change, exercises with the help of the swiss ball and electrotherapy. Final Considerations: The physiotherapeutic resources used offer positive results in reducing pain as well as the duration of labor, making it more active, humanized and satisfactory for the parturient.
\end{abstract}

Key words: Humanizing delivery, Physical therapy specialty, Women's health.

\section{RESUMEN}

Objetivo: Discuta las contribuciones de los recursos de fisioterapia aplicados en el trabajo natural humanizado. Métodos: Revisión de la literatura investigada en el horizonte temporal de 2009 a 2019 en las bases de datos electrónicas BVS, PubMed, SciELO, PEDro y Google Scholar. El proceso de análisis de los

${ }^{1}$ Centro Universitário UNIRB (FARAL), Alagoinhas - BA. *E-mail: carollinefisio@outlook.com 
artículos seleccionados se llevó a cabo mediante la lectura exploratoria y detallada de títulos, resúmenes y resultados de investigación, donde se buscaron las contribuciones de los recursos fisioterapéuticos aplicados en el trabajo natural humanizado. Resultados: Entre las opciones de los recursos fisioterapéuticos más utilizados para reducir el dolor en el proceso de trabajo natural humanizado se encuentran: ejercicios de respiración, terapia de masaje, termoterapia, cambio de posición, ejercicios con la ayuda de la pelota suiza y electroterapia. Consideraciones Finales: Los recursos de terapia física utilizados ofrecen resultados positivos en la reducción del dolor, así como la duración del trabajo de parto, haciéndolo más activo, humanizado y satisfactorio para el parto.

Palabras clave: Fisioterapia, Parto Humanizado, Salud de la Mujer.

\section{INTRODUÇÃO}

Em favor da humanização do parto, em 1980 ocorreu uma movimentação mundial preocupada em adotar novos paradigmas que considerassem e valorizassem o ser humano de forma global, e que excitassem os profissionais de saúde a repensarem sua prática. A avaliação científica das práticas notabilizou a eficácia e a segurança do parto com o mínimo de intervenção sobre a fisiologia e de procedimentos voltados para as necessidades da parturiente. Isto resultou em uma nova versão de assistência ao parto, denominado de humanista (OLIVEIRA VHB, et al.,2012).

O Ministério da Saúde explana o parto humanizado como aquele que tem a fisiologia respeitada, com mínimas intervenções necessárias, favorecimento aos desejos e necessidades da mulher e do bebê, presença de um acompanhante de sua livre escolha e práticas comprovadas cientificamente como benéficas para o binômio, tais como: aleitamento na primeira hora de vida, contato pele a pele precoce (golden hour), clampeamento de cordão após cessar a pulsação, entre outros (BRASIL, 2014).

Para o Ministério da Saúde a gestante pode contar com o suporte físico e emocional oferecido pelo seu acompanhante, doula (acompanhante especializado) e pelos profissionais que estão envolvidos com a assistência, entre estes o fisioterapeuta. Este profissional dispõe de recursos e conhecimentos cruciais para a orientação de posições, controle muscular e alívio de quadros álgicos durante o processo de trabalho de parto (BRASIL, 2014; CANESI K e AMARAL WN, 2010).

O profissional fisioterapeuta, segundo Silva SR, et al. (2012) e Freitas AS, et al. (2017) deve está inserido dentro da equipe multidisciplinar de atenção à mulher grávida, de modo que obtém recursos onde segundo Bavaresco GZ, et al. (2011) possibilitam um suporte eficiente e seguro que oferecem a subtração do quadro álgico durante todo o processo de trabalho e parto e parto natural.

O fisioterapeuta é apto a prestar assistência de modo ativo às parturientes, uma vez que possui recursos não farmacológicos que são capazes de atuar no quadro álgico das disfunções advindas do processo de parturição (SOUZA APK e RAMOS DJS, 2017). Diante do que foi exposto, questiona-se de que maneira estes recursos fisioterapêuticos podem contribuir no trabalho de parto natural humanizado?

A relevância deste trabalho é dada pela necessidade de debater e incentivar a adoção do parto natural humanizado considerando que os princípios da humanização priorizam os desejos e o protagonismo da mulher, juntamente com o fato de que este é o dinamismo de parto pelo qual o corpo feminino está preparado para passar, sendo um processo ativo, natural, saudável e de rápida recuperação. A atuação dos recursos fisioterapêuticos, neste processo torna-o menos doloroso e mais agradável para a parturiente, além de não farmacológico, de fácil acesso e baixo custo, não invasivo e do respeito ao mecanismo fisiológico do processo parturitivo natural humanizado.

Embasando-se nisto, este trabalho tem como objetivo discutir as contribuições dos recursos fisioterapêuticos aplicado no trabalho de parto natural humanizado. Espera-se que o presente estudo venha a colaborar no meio social, acadêmico e profissional levando maiores informações a cerca do assunto versado. 


\section{MÉTODOS}

Este trabalho se caracteriza como uma revisão de literatura, onde é utilizado o método dedutivo. Ainda sendo atribuído o rotulo de qualitativa, descritiva e exploratória à presente pesquisa. A coleta de material foi realizada em livros adquiridos através de indicações de profissionais fisioterapeutas e em bases eletrônicas de dados, tais como: Scielo, BVS, Pubmed, PEDro e Google Acadêmico.Para pesquisa dos materiais foram utilizados os seguintes descritores, previamente pesquisados no descritores em ciências da saúde (DESC): "Fisioterapia", "'Parto Humanizado", "Saúde da Mulher".

Como critério de inclusão foram aceitos artigos em idioma no português, inglês e espanhol, datados de publicação do ano de 2009 até novembro de 2019. Os demais artigos que não se enquadraram nos itens de inclusão foram excluídos, com exceção dos livros de autoria de Kitchen S (2003) e Low J e Reed A (2001).

\section{RESULTADOS}

Para a construção do presente artigo foi crucial o embasamento em estudos de casos, que demonstrassem o uso dos recursos fisioterapêuticos na atuação prática do profissional da fisioterapia. Deste modo, foram selecionados os estudos expostos no Quadro 1, onde pode-se visualizar os autores e os anos dos respectivos artigos publicados, bem como a especificação de quais dos os recursos foram utilizados no atendimento ao trabalho de parto natural humanizado e os principais resultados que foram alcançados na aplicação prática das terapias.

Quadro 1 - Resultados encontrados nos artigos selecionados

\begin{tabular}{|c|c|c|}
\hline Autor / Ano & Recursos Utilizados & Principais Resultados \\
\hline Gallo RBS, et al. (2018) & $\begin{array}{l}\text { Massoterapia; Termoterapia (Banho } \\
\text { quente); Exercícios com a bola } \\
\text { suíça. }\end{array}$ & $\begin{array}{l}\text { Redução do quadro álgico, do } \\
\text { tempo do trabalho de parto e } \\
\text { do uso de fármacos. }\end{array}$ \\
\hline Abreu NS, et al. (2013) & $\begin{array}{l}\text { Massoterapia; Alternância de } \\
\text { posições; Deambulação; Exercícios } \\
\text { Respiratórios. }\end{array}$ & $\begin{array}{l}\text { Diminuição da percepção } \\
\text { dolorosa e aumento da } \\
\text { segurança e conforto. }\end{array}$ \\
\hline Santos ERS e Oliveira C (2014) & $\begin{array}{l}\text { Exercícios Respiratórios; Exercícios } \\
\text { com a bola suíça. }\end{array}$ & $\begin{array}{l}\text { Aumento da tolerância da dor, } \\
\text { diminuição do desconforto e } \\
\text { do uso de analgésicos. }\end{array}$ \\
\hline Braz MM, et al. (2014) & Exercícios com a bola suíça. & Redução do quadro álgico. \\
\hline Castro AS, et al. (2012) & $\begin{array}{llr}\text { Exercícios } & \text { Respiratórios; } & \text { TENS; } \\
\text { Alternância de } & \text { Posições; } \\
\text { Massoterapia. } & & \end{array}$ & $\begin{array}{l}\text { Estabilização da dor, sendo } \\
\text { considerado um fator positivo } \\
\text { diante do fato de que nesta } \\
\text { fase a dor tende a aumentar. }\end{array}$ \\
\hline Barros AP e Matos SS (2017) & $\begin{array}{l}\text { Massoterapia; Exercícios } \\
\text { Respiratórios; Alternância de } \\
\text { Posições; Termoterapia (Banho } \\
\text { Quente). }\end{array}$ & $\begin{array}{l}\text { Redução do quadro álgico e } \\
\text { aumento da confiança e } \\
\text { segurança da parturiente. }\end{array}$ \\
\hline Gallo RBS, et al. (2014) & Exercícios com a bola suíça & Redução do quadro álgico. \\
\hline
\end{tabular}

Fonte: Cruz CB, et al., 2020.

\section{DISCUSSÃO}

Yuksel H, et al. (2017) entra em acordo com Gallo RBS, et al. (2018) e Abreu NS, et al. (2013) quando afirma que a realização exercícios respiratórios de inspiração e expiração profunda desde os $4 \mathrm{~cm}$ de dilatação cervical, durante o processo de trabalho de parto contribui positivamente com a redução da percepção de dor e a diminuição do tempo do trabalho de parto. Pois segundo Batista PCA, et al. (2012), o corpo feminino passa por alterações compensatórias na tentativa de manter a homeostase materno-fetal influindo na saturação de oxigênio acarretando desconfortos respiratórios e os exercícios respiratórios têm ação direta nestas mudanças através da expansão pulmonar como uso da inspiração e expiração profunda. 
Castro AS, et al. (2012) e Barros AP e Matos SS (2017) corroboram com Gallo RBS, et al. (2018), quando obtiveram resultados positivos com parturientes através da implementação de recursos fisioterapêuticos com a utilização dos exercícios respiratórios de expansão pulmonar melhorando a saturação de oxigênio e reduzindo as ações compensatórias do mecanismo de homeostase do processo de gestação. Para Sousa VPS, et al. (2011) e Silva JL, et al. (2010), o útero gravídico é o principal motivo das ações compensatórias pois o mesmo está continuamente em crescimento e conseguinte de um órgão pélvico o útero se expande em elevação e torna-se um órgão abdominal, aumentando 150 vezes a dimensão e mais de mil vezes a capacidade no processo da gestação.

Sousa CB, et al. (2018) expõe que quando a mãe realiza uma inspiração expandindo a parede abdominal, abaixa o diafragma, potencializando o poder de ação do músculo que estava comprimido pela elevação do útero gravídico, assim aliviando as algias, favorecendo o relaxamento e a oxigenação do binômio mãe-bebê, beneficiando a concentração e reduzindo as chances de traumas perineais na mãe.

Em um estudo realizado por Kamalifard $M$, et al. (2012) utilizaram de técnicas de respiração e da massoterapia com o intuito de alcançar a redução da dor e das respostas fisiológicas no trabalho de parto. O trabalho supracitado abordou que a utilização dos dois recursos de modo conjunto ou singular trouxe benefício ao objetivo proposto, obtendo eficácia desde os $4 \mathrm{~cm}$ de dilatação cervical. Além disto, o artigo ainda afirma que foi possível reduzir as taxas de parto cesáreo, corroborando com os autores Sousa CB, et al. (2018) e Gallo RBS, et al. (2013).

Além dos exercícios respiratórios pode-se optar pelo uso da massoterapia onde Gallo RBS, et al. (2013) entrou em comunhão com Castro AS, et al. (2012) e Abreu NS, et al. (2013) quando afirma que por meio da aplicação do recurso fisioterapêutico da massagem na fase ativa do trabalho de parto natural desde os $4 \mathrm{~cm}$ de dilatação cervical, evidenciou redução da intensidade do quadro álgico. Ferreira ACM e Martins HO (2013) aborda que a amenização da algia é possível, pois o recurso de massoterapia possibilita a melhora da circulação sanguínea, mobilidade dos tecidos e do segmento.

A massoterapia oferece a ampliação do suplemento sanguíneo e demanda de oxigênio nas áreas tratadas, diante disto ocasiona a redução do quadro álgico e do estresse. A pressão exercida comprime os tecidos moles e estimula os receptores sensoriais, proporcionando sensação de prazer e bem-estar, juntamente com a melhora da qualidade da pineal e da qualidade de vida (ABREU MF, et al., 2012).

É importante ressaltar que a diminuição dos níveis de estresse previne a hiperventilação, onde por sua vez, há a prevenção da alcalose respiratória, o quê restringe a catecolaminas, contribuindo assim para baixas incidências de acidose fetal. Desta maneira, a redução do estresse acarreta positivos resultados para neonatais. Tendo isto em vista, a massoterapia contribui não apenas com a melhora do quadro álgico e do estresse no momento do trabalho de parto natural humanizado, como também no momento pós parto, além de influenciar positivamente o neonato e não apenas a parturiente (GAYESKI ME e BRÜGGEMANN OM, 2010).

Gallo RBS, et al. (2013) corrobora com Gallo RBS, et al. (2018) e Barros AP e Matos SS (2017) finalizando os seus respectivos estudos com uma visão positiva da aplicabilidade do recurso de massoterapia no trabalho de parto. Conforme Ferreira ACM e Martins HO (2013) a utilização do recurso melhora a oxigenação, nutrição e chegada de substâncias do produzidas pelo organismo que trazem melhoras ao quadro álgico, juntamente com a otimização da circulação sanguínea, assim como a mobilidade permite que ocorra uma dilatação menos dolorosa devido a maior flexibilidade proporcionada.

Benfield RD, et al. (2010) em anuência com Gallo RBS, et al. (2018) conseguiu resultados benéficos com a subtração do quadro álgico em mulheres durante a fase ativa do trabalho de parto com o uso do recurso da termoterapia através do banho quente $\left(37^{\circ} \mathrm{C}\right)$. Este resultado na visão de Lee LS, et al. (2013), pode ser contemplado a partir do momento que o estimulo da dor atravessa os receptores da coluna vertebral para o cérebro onde se sucede a resposta de modo direto, por tanto o banho quente estimula termorreceptores localizados na epiderme que chegam ao cérebro antes que os estímulos enviados pelos receptores da algia, bloqueando de maneira efetiva a transmissão, ou seja a percepção da dor. 
Em harmonia com Lee LS, et al. (2013), Benfield RD, et al. (2010) afirma que o banho quente promove uma estimulação cutânea de calor superficial que em somatória com a intensidade determinada e com o período de aplicação, oferece efeitos sistêmicos e gerais, que contribuem para a redução do quadro álgico. Ainda segundo Lee LS, et al. (2013) há duas maneira de utilizar a termoterapia através do banho quente, sendo ela o banho de aspersão e o banho de imersão, onde ambas devem estar a $37^{\circ} \mathrm{C}$, durante 0 processo de trabalho de parto.

Barros AP e Matos SS (2017) também obtiveram resultados positivos na redução do quadro álgico em parturientes desde os $4 \mathrm{~cm}$ de dilatação cervical, com o uso do banho quente (37으). Lee LS, et al. (2013) justifica que esse efeito é possível devido a ação do calor na circulação sanguínea acalmando o stress proporcionado pelas contrações, melhora do metabolismo e da elasticidade. Benfield RD, et al. (2010) agrega mais informações sobre o efeito do banho quente sob o quadro álgico quando afirma que o recurso também tem resultados positivos por afetar as respostas neuroendócrinas que modificam os processos psicofisiológicos.

Diante dos resultados positivos encontrados por Gallo RBS, et al. (2018), quanto a diminuição da percepção dolorosa no trabalho de parto, através do uso da termoterapia, Benfield RD, et al. (2010) explana que o banho quente seja de aspersão ou de imersão, oferece a parturiente efeitos positivos da higiene física, além de inúmeros benefícios e diferenças significativas no processo de trabalho de parto natural humanizado, tendo em vista que o mesmo otimiza a circulação sanguínea através da vasodilatação, influenciando positivamente na oxigenação e na chegada de substancias essenciais ao organismo para a diminuição da percepção dolorosa.

Taavoni S, et al. (2016) em assenso com Gallo RBS, et al. (2018), Barros AP e Matos SS (2017) e Abreu NS, et al. (2013) optou pela utilização em conjunto de dois recursos fisioterapêuticos distintos em seu estudo, sendo estes o de termoterapia com a aplicação da técnica de banho quente e exercícios cinesioterapêuticos realizados com o auxilio da bola suíça. Ambos os autores concluíram que a utilização em conjunto das duas técnicas trouxe a diminuição da algia, tendo em vista que o banho quente potencializa o efeito da bola suíça e vice e versa.

Leung RW, et al. (2013) encontrou resultados positivos quanto ao uso da bola suíça, sendo este a redução do quadro de algia bem como do uso de petidina por mulheres em trabalho de parto natural. Segundo Gallo RBS, et al. (2014), a bola suíça beneficia a evolução positiva da dilatação cervical durante todo o processo do trabalho de parto natural humanizado, dessa maneira, torna-se possível que ocorra a diminuição do tempo de parto, bem com da dor e da necessidade do consumo de fármacos pelas parturientes.

Em um estudo randomizado estruturado em Tawian de autoria de Gau ML, et al. (2011), os resultados se tornaram compatíveis com os encontrados por Gallo RBS, et al. (2014) e Braz MM, et al. (2014) ao utilizarem a cinesioterapia através da bola suíça, desde os $4 \mathrm{~cm}$ de dilatação cervical, onde a bola encoraja a parturiente a assumir posições alternadas e verticalizadas, apresentando diminuição no quadro álgico e no tempo do trabalho de parto.

Zwelling E (2010) explana que posições verticalizadas permitem que a força da gravidade juntamente com o alinhamento do eixo fetal com a bacia materna, beneficie a descida e a progressão fetal no canal de parto, tento isto em vista a posição e a movimentação da parturiente ao decorrer do trabalho de parto gera a diminuição da percepção de dor, privilegia a circulação materno-fetal, reduz o tempo do trabalho de parto e influenciam na queda das taxas de trauma perineal e de episiotomia.

A eletroterapia também está inclusa no leque de opções dos recursos fisioterapêuticos, destacando-se o aparelho de estimulação elétrica transcutânea nervosa (TENS), usado por Santana LS,et al. (2016) que em congruência com Abreu EA, et al. (2010) lograram melhora significativa da dor, desde os $4 \mathrm{~cm}$ de dilatação cervical. Segundo Kitchen S (2003), a atuação da TENS ocorre através da teoria das comportas da dor, onde essas comportas são fechadas pela estimulação da corrente elétricas das fibras A $\beta$ que fazem parte do segmento espinal, sendo estes os mesmos que as fibras nociceptivas relacionadas com a sensação da 
dor, desta forma quando o estimulo atinge o cérebro fechando as comportas e impedindo que a informação nociva atinja o cérebro consequentemente ocorre a redução da sensação de dor.

Castro AS, et al. (2012), entra em conformidade com Abreu EA, et al. (2010) ao alcançar resultados positivos na atenuação do quadro álgico em parturientes no trabalho de parto natural. Bavaresco GZ, et al. (2011) explana que a estimulação elétrica transcutânea proporciona analgesia uma vez que atua na ativação de receptores sensoriais elevando os níveis de endorfina e como consequência reduzindo o quadro álgico.

Um estudo de Cappeli AJ (2018), demonstrou que através do uso da TENS na fase ativa do trabalho de parto, tornou-se possível à diminuição da intensidade da dor e a otimização do grau de desconforto após o uso da intervenção. Tendo isto em vista, a autora, afirma que o recurso apresentou eficácia ao decorrer do trabalho de parto, dando destaque a ação do mesmo no primeiro período do processo parturitivo. Desta maneira entra em concordância com o estudo de Abreu EA, et al. (2010), que afirma que o uso da TENS acarreta diminuição do quadro álgico, porém não há efeito sobre o tempo de parto.

Low J e Reed A (2001) retrataram que o efeito da estimulação elétrica transcutânea de alta frequência e baixa intensidade é decorrente da estimulação de fibras mecanorreceptores ( $A$ beta), que através do corno posterior influencia nas fibras $\mathrm{A}$ delta (dor rápida) e $\mathrm{C}$ (dor lenta), desta forma fechando as comportas da dor e reduzindo significativamente o quadro álgico. A excitação das fibras $A$ delta e $C$ proporciona $o$ efeito morfina devido a encefalina (neurotransmissor narcótico secretado pelo encéfalo) presente no sistema de fibras C, produzida no corno posterior que é estimulado pelas fibras A delta, assim como envolve a serotonina, sendo esta um neurotransmissor também presente no sistema de fibras $C$ e estimulada pelas fibras A delta, porém ocorre por meio de centros no mesencéfalo.

Abreu NS, et al. (2013) acorda com Santos ERS e Oliveira C (2014), e Barros AP e Matos SS (2017), quando concluem que a deambulação e a alternância de posições são benéficas a parturiente com a diminuição do quadro álgico e do tempo de trabalho de parto. Visto que Silva DAO, et al. (2013) afirma que alternância de posições usando-se de sedestração, deambulação, ortostase, com o apoio nos joelhos e de quatro apoios, auxilia no alivio da algia em razão da ação da gravidade que melhora a chegada do fluxo sanguíneo ao bebê através da placenta e das mudanças no formato da pelve, a movimentação auxilia na eficácia da contração, o parto torna-se mais curto e menos doloroso.

Foi abordado em um estudo de autoria de Nieuwenhuijze $\mathrm{H}$, et al. (2012) que mulheres que assumiram diversas posições durante $\mathrm{o}$ trabalho de parto obtiveram uma redução significativa no quadro álgico, bem como no tempo de trabalho de parto, ainda dando destaque a preferencia das parturientes a assumir posições eretas, onde há a atuação da gravidade como benéfico a progressão fetal, que influência diretamente tanto na álgia quanto no tempo de parto. Desta forma, Nieuwenhuijze H, et al. (2012) também destaca a satisfação das parturientes com o recurso de mudanças de decúbito como positivo ao processo.

Em um estudo realizado por Lawrence A, et al. (2013) tornou-se possível observar que há resultados positivos com o uso de alternância de posições por mulheres em processo parturitivo, dando destaque para posições eretas, onde demonstrou redução no tempo do parto e na necessidade de uso de parto cesáreo e da peridural. Nieuwenhuijze $\mathrm{H}$, et al. (2012) ainda expõe que os resultados positivos se tornaram possíveis diante do auxílio oferecido pela gravidade que exerce uma força vertical agregando na progressão do fetal pelo canal vaginal e contribui afirmando que as mudanças de posições devem ser encorajadas.

Lawrence A, et al. (2013) acredita que há evidencias onde a deambulação e as posturas verticalizadas durante o processo parturitivo estão associadas à redução do tempo do trabalho de parto e sem uma necessidade de uma maior intervenção ou efeitos negativos ao bem-estar do binômio mãe-bebê, portanto a autora acredita que as mulheres devem ser encorajadas a adotar posições mais confortáveis ao processo de parto. Uma vez que Bavaresco GZ, et al. (2011) afirma que posições como ajoelhada, sedestração, ortostase com inclinação de tronco, posição de Simms ou de cócoras sustentada e quatro apoios, diminuem a duração do trabalho de parto, aumenta a tolerância a dor, evita a fadiga, diante do fato que auxiliam o encaixe do feto a pelve materna e ao canal de parto. 


\section{CONSIDERAÇÕES FINAIS}

Os recursos fisioterapêuticos são aptos a contribuir no trabalho de parto natural humanizado através da subtração do quadro álgico, diminuindo ou até abolindo a utilização de fármacos e/ou técnicas que sejam invasivas e/ou desnecessárias, além de reduzir o tempo de duração do trabalho de parto. No decorrer da construção deste trabalho houve dificuldades para encontrar estudos que realizados por profissionais da área de fisioterapia e por tanto, estudos voltados para a área de obstétrica com foco na atuação do profissional fisioterapeuta devem ser estimulados, obtendo em vista também a dificuldade para encontrar estudos sobre o tema que se adequassem no horizonte temporal de 10 anos, principalmente que abordassem a via de ação da TENS.

\section{REFERÊNCIAS}

1. ABREU EA, et al. Efetividade da eletroestimulação nervosa transcutânea no alivio da dor durante o trabalho de parto: um ensaio clinico controlado. Revista Dor, 2010; 11(4): 313-318.

2. ABREU MF, et al. Os Efeitos da massoterapia sobre o estresse físico e psicológico. Revista Científica da Faculdade de Educação e Meio Ambiente. Blumenau, 2012; 3(1): 101-105.

3. ABREU NS, et al. Atenção fisioterapêutica no trabalho de parto e parto. Revista interdisciplinar de estudos experimentais, 2013; 5(1): 7-15.

4. BARROS AP, MATOS SS. A Importância da Atuação do Fisioterapeuta no Parto Vaginal em Primigestas e Multíparas. Revista Científica Multidisciplinar Núcleo doConhecimento, 2017; 1(1): 282-291.

5. BATISTA PCA, et al. Repercussão ventilatória impostas pelo ciclo gestatório: Uma abordagem fisioterapêutica sobre a saturação do oxigênio. . Suplemento Fisioterapia Brasil - I Encontro Nordestino de Fisioterapia em Saúde da Mulher - ENFISM. Natal - RN, 2012; 13(6): 171-175.

6. BAVARESCO GZ, et al. O fisioterapeuta como profissional de suporte à parturiente. Ciência\&SaúdeColetiva, 2011; 16: 3259-3266.

7. BENFIELD RD, et al. The Effects of Hydrotherapy on Anxiety, Pain, Neuroendocrine Responses, and Contraction Dynamics During Labor. Biol Res Nurs, 2010; 12(1): 28-36.

8. BRASIL. Ministério da Saúde. Caderneta da Gestante. Brasília - DF. 2014.

9. BRASIL. Ministério da Saúde. Caderno do HumanizaSUS: Humanização do Parto ao Nascimento. Volume 4. Brasília - DF. 2014.

10. BRAZ MM, et al. Bola do Nascimento: Recurso fisioterapêutico no trabalho de parto. Cinergis, 2014; 15(4):168-175.

11. CANESIN K, AMARAL WN. Atuação fisioterapêutica para diminuição do tempo do trabalho de parto: revisão de literatura. Femina, 2010; 38(8): 429-433.

12. CAPPELI, AJ. Estudo randomizado do uso da estimulação elétrica nervosa transcutânea (TENS) no alívio da dor no trabalho de parto. 2018.

13. CASTRO AS, et al. Abordagem fisioterapêutica no pré-parto: proposta de protocolo e avaliação da dor. Fisioterapia e Pesquisa, 2012; 19(3): 210-214.

14. FERREIRA ACM, MARTINS HO. Movimentação passiva continua: Conceito e aplicação. Caderno de Estudos Tecnologicos, 2013; 1(1): 177-188.

15. FREITAS AS, et al. Atuação da fisioterapia no parto humanizado. DêCiência em Foco, $2017 ; 1(1)$.

16. GALLO RBS, et al. A bola suiça no alivio da dor de primigestas na fase ativa do trabalho de parto. Rev. dor, 2014; 15(4): 253-255.

17. GALLO RBS, et al. Massage reduced severity of pain during labour: a randomized trial. Journal of Physiotherapy, 2013; 59(2): 109-116.

18. GALLO RBS, et al. Sequential application of non-pharmacological interventions reduces the severity of labour pain, delays use of pharmacological analgesia, and improves some obstetric outcomes: a randomised trial. Journal os Physiotherapy, 2018; 64(1): 33-40.

19. GAU ML, et al. Effects of birth ball exercise on pain and self-efficacy during childbirth: a randomised controlled trial in Taiwan. Midwifery, 2011; 27(6): 293-300.

20. GAYESKI ME, BRÜGGEMANN OM, Métodos não farmacológicos para alívio da dor no trabalho de parto; uma revisão sistemática. Texto Contesto Enferm, 2010, 19(4): 774-782.

21. KAMALIFARD M, et al. The Efficacy of Massage Therapy and Breathing Techniques on Pain Intensity and Physiological Responses to Labor Pain. J Caring Sci, 2012; 1(2): 73-78.

22. KITCHEN S. Eletroterapia prática baseada em evidência. 2ª Edição. Barueri - São Paulo: Manole, 2003. 
23. LAWRENCE A, et al. Maternal positions and mobility during first stage labour. Cochrane Database of Systematic Reviews, 2013, 20(8).

24. LOW J, REED A. Eletroterapia explicada: Principos e prática. 3ª Edição. Barueri - São Paulo: Manole, 2001.

25. LEE SL, et al. Efficacy of Warm Showers on Labor Pain and Birth Experiences During the First Labor Stage. Jognn, 2013; 42: 19-28.

26. LEUNG RW, et al. Efficacy of birth ball exercises on labour pain management. Hong Kong Med J, 2013, 19(5): 393399.

27. NIEUWENHUIJZE M, et al. Factors influencing the fulfillment of women's preferences for birthing

28. positions during second stage of labor. Journal of Psychosomatic Obstetrics \& Gynecology, 2012, 33(1): 25-31.

29. OLIVEIRA VHB, et al. Recursos fisioterapêuticos disponíveis para trabalho de parto: uma revisão. Suplemento Fisioterapia Brasil - I Encontro Nordestino de Fisioterapia em Saúde da Mulher - ENFISM. Natal - RN, 2012; 13(6): 16-22.

30. SANTANA LS, et al. Transcutaneous electrical nerve stimulation (TENS) reduces pain and postpones the need for pharmacological analgesia during labour: a randomised trial. JournalPhysitherapy, 2016; 62: 29-34.

31. SANTOS ERS, OLIVEIRA C. Influência da cinesioterapia na fase ativa do trabalho de parto no centro de pré-parto, parto e pós parto do instituto da mulher Dona Lindu. 2014.

32. SILVA DAO, et al. Uso de métodos não farmacológicos para o alívio durante o trabalho de parto normal: revisão integrativa. Revista de Enfermagem, 2013; 7: 4161-70.

33. SILVA JL, et al. Orientações pré-natais e a influência na qualidade de vida de gestantes saudáveis. Fisioterapia Brasil, 2010; 11(2).

34. SILVA SR, et al. Influência da cinesioterapia sobre a dor e qualidade de vida em mulheres grávidas. Suplemento Fisioterapia Brasil - I Encontro Nordestino de Fisioterapia em Saúde da Mulher - ENFISM. Natal - RN, 2012; 13(6): 23-28.

35. SOUSA CB, et al. Atuação da Fisioterapia para a redução do tempo no trabalho de parto vaginal. ScierSalutis, 2018; 8(2): 123-128.

36. SOUSA VPS, et al. Analiseda relação entre dor lombar, idade gestacional e qualidade devida durante a gravidez. Terapia Manual, 2011; 9(46): 835-40.

37. SOUZA APK, RAMOS DJS. Fisioterapia e humanização do parto: Uma análise partir de documentos oficiais da saúde. Revista Fisioterapia Reabilitação, 2017; 1(1): 11-23.

38. TAAVONI S, et al. Birth ball or heat therapy? A randomized controlled trial to compare the effectiveness of birth ball usage with sacrum-perineal heat therapy in labor pain management. Complementary Therapies in Clinical Practice, 2016; 24: 99-102.

39. YUKSEL $\mathrm{H}$, et al. Effectiveness of breathing exercises during the second stage of labor on labor pain and duration: a randomized controlled trial. Journal of Integrative Medicine, 2017; 15(6): 456-461.

40. ZWELLING E. Overcoming the challenges: maternal movement and positioning to facilitate labor progress. MCN Am J Matern Child Nurs, 2010; 35(2): 72-80. 